\title{
Classical Trajectory Monte Carlo Code For Simulating Ion Beam Focusing Or Defocusing With Magnetic Elements Modeled As Current Loops Or Current Lines
}

\author{
R. A. Lane and C. A. Ordonez \\ Department of Physics, University of North Texas, Denton, Texas
}

\begin{abstract}
A computational tool is described that can be used for designing magnetic focusing or defocusing systems. A fully three-dimensional classical trajectory Monte Carlo simulation has been developed. Ion trajectories are simulated in the presence of magnetic elements that can be modeled as any combination of current loops and current lines. Each current loop or line may be located anywhere in the system and oriented along any of the three coordinate axes. The configuration need not be axisymmetric. The solutions are obtained using normalized parameters, which can be used for easily scaling the results. Examples are provided of the utility of the code.
\end{abstract}

Keywords: Mathematica, Monte Carlo, Magnetic Lens, Plasma Trap

PACS: 52.55.Dy, 52.65.Cc, 52.65.Pp, 41.85.Lc

\section{INTRODUCTION}

We have developed a generalized program in Mathematica [1] for conducting classical trajectory Monte Carlo (CTMC) simulations of ion motion within a magnetic field. The program allows the user to construct magnetic lenses and plasma traps from any number of current loops and current lines. It measures the particle loss from the system and simulated strikes on detectors for a range of particle initial positions and current strengths. The program is automated to incrementally test many parameter sets, and thus can be used for optimizing novel designs.

For the CTMC simulations, the paths of many particles are tested with randomized particle initial velocities. The user configures the position and orientation of the current lines and loops, detectors, and system boundaries, the shape and limits of the velocity distribution, and the initial position(s) of the particles.

The program allows the user to tailor almost all of the characteristics of the simulation and the automation tests as wide or narrow a range of the key parameters (current, particle initial position, sample size, maximum particle time of flight) as desired. The variability of the program parameters and the system configuration allows this program to be used to compare a wide variety of systems quickly and efficiently with minimal user interaction or to carefully examine how the capabilities of a single system change under diverse parameters.

The program focuses on calculating the end location statistics (detector strikes and fraction of particles which escape the system) for customizable magnetic field configurations. This allows flexibility in applications. It can be used to simulate ion beam interactions with focusing and defocusing magnetic lenses as well as plasma confinement in traps, for which CTMC simulation studies have proven effective (for examples, see: [2] and [3]).

There are commercially available programs for simulating ion optics such as SIMION [4]. Our program differs in that it is written for Mathematica and that its focus is simulating the interaction of particles with generic magnetic fields. The simulation is written for a widely used software package with built-in parallel processing tools and can automatically distribute jobs over multiple processing nodes on a supercomputer.

The advantage of this program being written as a Mathematica script is two-fold. All of the system variables, equations, and data variables are in the Mathematica notebook at the end of the simulation. The user can work with these variables to do individual calculations. The availability and high level of Mathematica easily allow the user to tailor the program to suit individual needs. These advantages give our program great flexibility in application.

Application of Accelerators in Research and Industry

(C) 2013 AIP Publishing LLC 978-0-7354-1148-7/\$30.00 


\section{PROGRAM DESCRIPTION}

Our computational program calculates and monitors the trajectory of particles in a customizable magnetic field in normalized, dimensionless parameter space to obtain generalized results. It calculates the fractions of particles that reach user-designated locations within the system (detectors) or the edge of the system during a given time frame and automatically increments the sample size and maximum particle flight time to find consistent results.

The magnetic field everywhere in space is automatically constructed from the user-specified locations, orientations, and current strengths of the current loops and lines. Each element may be placed anywhere in space and may be oriented along any of the three coordinate axes. Also, the currents may be specified relative to one another or defined independently. The magnetic field of a current line everywhere in space is well known and its derivation is a common student assignment (see, for example, [5] or [6]). However, the magnetic field of a current loop is more complicated, but has been calculated using multiple methods (see [7], [8], and [9]). As a matter of preference, we choose to use the elliptic integral forms calculated in [7]. The program automatically applies standard rotations and linear shifts to orient the elements relative to one another.

The program starts each simulated particle from a user supplied initial position and gives the particle a randomized initial heading with its directions specified by the azimuthal and altitudinal angles, $\varphi_{0}$ and $\theta_{0}$, respectively. The angles may take any value with $0 \leq$ $\varphi_{0}<2 \pi$ and $0 \leq \theta_{0} \leq \theta_{\max }$, where $\theta_{\max }$ can take any userspecified value in the range $0<\theta_{\max } \leq \pi$. Also, the particles may be mono-energetic or follow a Gaussian velocity distribution. The program uses the initial conditions and the calculated total magnetic field to find the equations of motion for the particle. The equations of motion are solved as functions of normalized, dimensionless time $\left(t_{n}\right)$.

The paths of the particles are checked for special conditions from $t_{n}=0$ to an incrementally varied maximum time $\left(t_{n, \max }\right)$. The single particle simulation is terminated at less than $t_{n, \max }$ if the particle reaches either the edge of the system or a simulated detector. If it reaches the edge of the system it is recorded as having escaped, while if it reaches a detector, the program records the time of the strike and the detector location. The edge of the system is defined by the user, and can be the inner surface of a cube, cylinder, or sphere of customizable dimensions and orientation, which encloses all system components. A user may also specify any number of simulated detectors, which may be cubes, cylinders, or spheres with any size and orientation.

The program includes the ability to automatically increment many system parameters to minimize required user interaction. It can increment or try a specified set of values for the current(s) and the initial particle positions. The currents of the components can be varied individually or together. A similar process is used to incrementally check a variety of sample sizes and $t_{n, \max }$ values for each initial position to insure that the particle paths are not being ended prematurely or are under sampled. To decrease processing time, the sample size and $t_{n, \max }$ values start at a specified minimum and increase until the desired consistency between runs is reached. The increments cannot increase without bound and will stop after a preset number of iterations. Non-convergent data is flagged appropriately but the information for the last iteration is logged to prevent missing data points.

\section{APPLICATIONS}

The primary application of the CTMC simulation program described above is to test the interaction of diffuse plasmas and ion beams with novel magnetic field configurations, neglecting particle interactions. Two key types of information are obtained, the particle end location statistics and the path of each particle through the system.

When averaged over a sufficiently large number of randomized particles, this information gives a picture of how charged particles will move and interact with the system. This information can be directly used in studies of focusing or defocusing using multipleelement magnetic lenses and studies of plasma confinement in novel confinement systems. The program may also be used as a teaching tool to generate figures and information for students of plasma confinement, accelerator design, and those first encountering particles moving in magnetic fields.

The program easily lends itself to testing the focusing or defocusing power of magnetic lenses. Many types of two- and three-dimensional magnetic lenses may be modeled from combinations of current loops and lines. The limits on the initial position and/or velocity settings of the sample set can be used to model as diffuse or focused an initial beam as desired. The simulated detectors may be organized in a tight grid pattern at the opposing side of the lens in order to measure distribution of the transmitted beam.

The CTMC simulations can also be used to test for plasma confinement within novel magnetic confinement systems. The program allows the user to find the fraction of particles that escape the system as a function of the magnetic field strength and the initial 
position of the particles by varying the system parameters incrementally.

\section{EXAMPLE SIMULATIONS}

The simulation program can be used to test the converging or diverging power of novel magnetic lenses. For example, consider the lens created by two current lines equidistant from the origin along the $x$ axis and extending to infinity along the $y$-axis, see Fig. 1. For this simulation we assume that the particles start at $(0,0,-3)$, are mono-energetic, and their heading has coordinates $0 \leq \varphi_{0}<2 \pi$ and $0 \leq \theta_{0} \leq \pi / 15$. To test the distribution of the particles a grid of 400 square detectors with sides of length 0.07 normalized units is placed with its center at $(0,0,3)$.

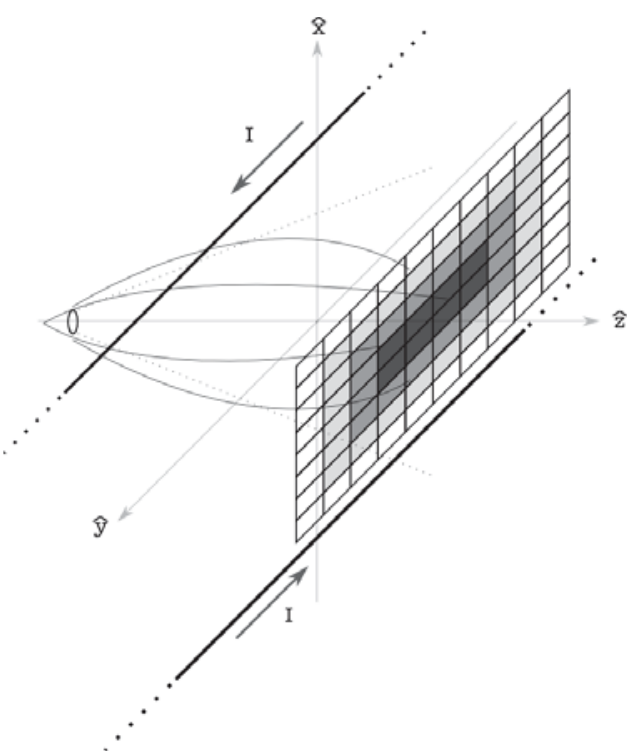

FIGURE 1. An example of a focusing magnetic lens with two current lines that extend to positive and negative infinity in the $y$ direction and are equidistant from the origin. Particles are incident from the position $(0,0,-3)$ and are focused by the lens. The transmitted particle distribution is measured with a rectangular array of detectors.

The paths of 75 simulated particles moving through the converging lens are shown for four different values of magnetic field strength in Fig. 2. In Fig. 3, the distribution of the particles at the detector grid is shown. The information displayed in these two figures can be combined to calculate the defining characteristics of the lens and transmitted beam. The automation of the program easily facilitates calculating how these characteristics change with magnetic field strength and initial particle position.

The program also has the ability to model the dispersing effects of a magnetic configuration on an initially tight particle distribution. The initial beam may be modeled as incident from a single initial position with its range about the $z$-axis limited or can be modeled as a tight grouping of initial positions. As in the converging case, the distribution and dispersion of the particles can be examined. This feature can be used to model and reduce the undesired effects of experimental components. It can also be used in combination with converging elements and detectors to model and design ion beam lines. An example of this feature using a mono-energetic beam incident from $(0,0,-3)$ with its initial velocity having $0 \leq \theta_{0} \leq \pi / 60$, traveling through a current loop centered on the origin and striking a detector grid at $(0,0,3)$, is shown in Fig. 4.

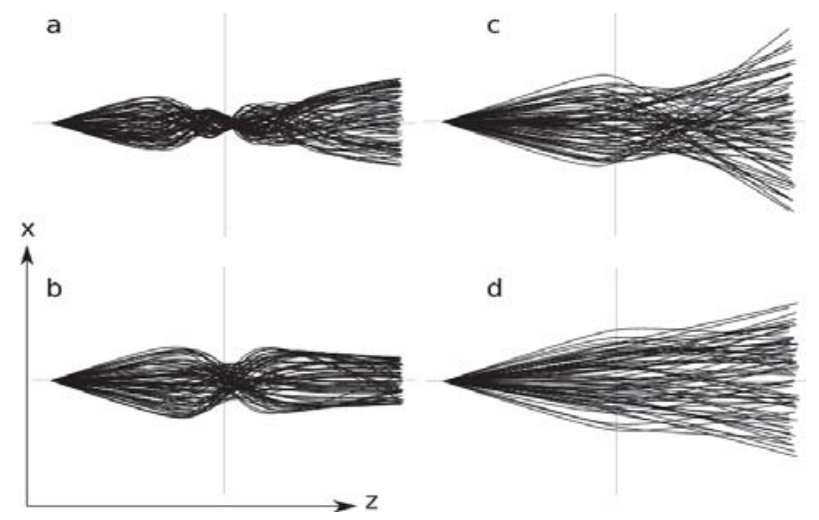

FIGURE 2: The 2D paths of 75 simulated particles through the system pictured in Fig. 1 are shown. The magnetic field strength is decreased by a factor of 2 from (a) to (b), (b) to (c), and (c) to (d).

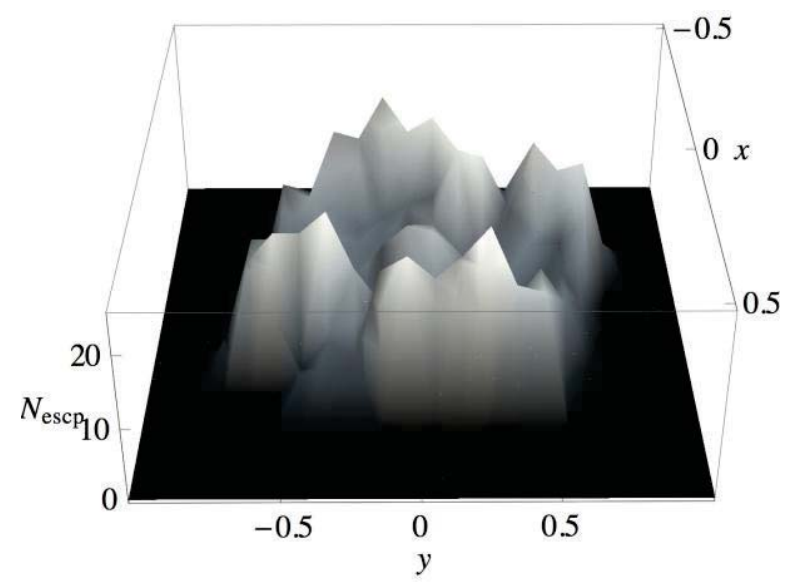

FIGURE 3: The distribution of particle strikes on the detector grid at $(0,0,3)$ in the system from Fig. 1, is shown for 1000 incident particles.

The program's applications are not restricted to ion beam optics. It can also be used to simulate the interaction of plasma with magnetic fields. Many 
applications of plasma physics depend on the ability to confine plasma. The simulation may be used to model the confinement capabilities of novel systems quickly and efficiently. As an example, consider the system from [10], which consists of two concentric current loops separated by a distance smaller than the loop radius. The fraction of particles which escape to the system boundaries is sampled in a grid pattern with 0.7 $\leq \mathrm{x}_{\mathrm{n}, 0} \leq 1.2$ and $0 \leq \mathrm{z}_{\mathrm{n}, 0} \leq 0.2$. The resulting data can be used to find the regions of initial particle positions where the plasma could be confined as a function of current, as shown in Fig. 5.

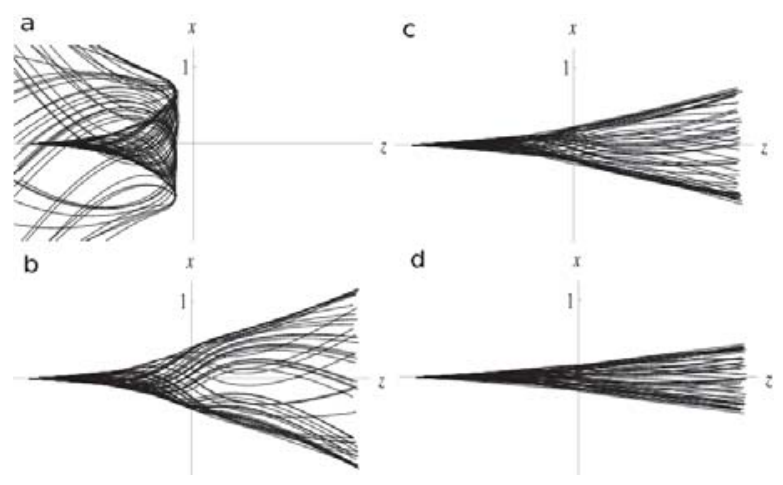

FIGURE 4: The 2D paths of 75 particles, incident from the left, passing through a current loop with a radius of unity, and centered at the origin, and striking a square detector grid (not shown) that lies in the $x-y$ plane and is centered on the $z$ axis. The paths are shown for four values of magnetic field strength. The magnetic field strength is decreased by a factor of 2 from (a) to (b), (b) to (c), and (c) to (d).

The program's applications are not restricted to ion beam optics. It can also be used to simulate the interaction of plasma with magnetic fields. Many applications of plasma physics depend on the ability to confine plasma. The simulation may be used to model the confinement capabilities of novel systems quickly and efficiently. As an example, consider the system from [10], which consists of two concentric current loops separated by a distance smaller than the loop radius. The fraction of particles which escape to the system boundaries is sampled in a grid pattern with 0.7 $\leq \mathrm{x}_{\mathrm{n}, 0} \leq 1.2$ and $0 \leq \mathrm{z}_{\mathrm{n}, 0} \leq 0.2$. The resulting data can be used to find the regions of initial particle positions where the plasma could be confined as a function of current, as shown in Fig. 5.

The detectors may be used to simulate electrically neutral objects used to physically support components that will be used in a laboratory experiment. In this way the detector function can be used to calculate the fractional loss of particles to the wires used to provide current to the loops or lines as well as rods used to keep the loops in place. Both the current lines and loops as well as multiple detector configurations can be used to simulate complicated systems or even simple beam lines and to find the distribution of particles at the target location, the fractional loss of particles due to dispersion, and losses due to collisions with the support structures.

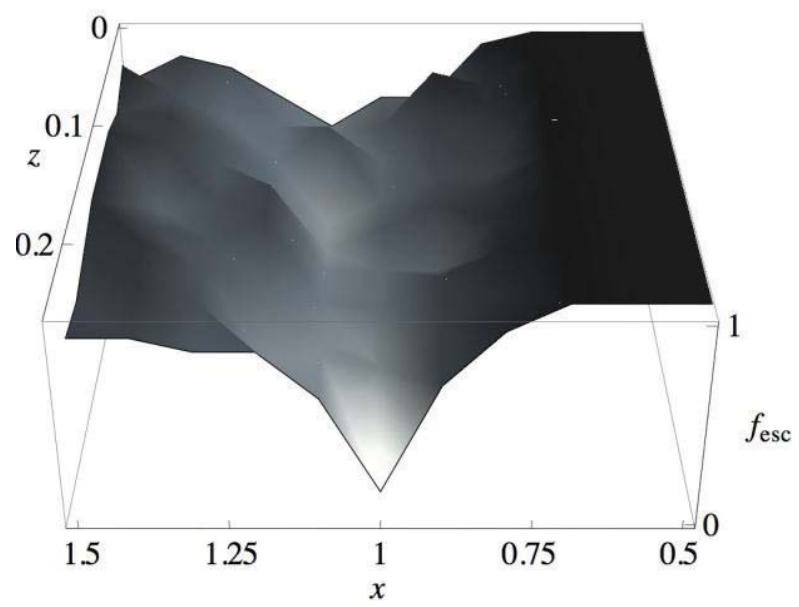

Figure 5: Two current loops with radius unity carrying the same current are centered on the z-axis 0.25 normalized units from the origin. The fraction of particles which escape from this system is shown for 96 different initial particle positions with a first order interpolation used to create a smooth surface.

\section{CONTINUING WORK}

Work on this program is continuing. The program will be expanded to allow the elements to take any orientation and to specify system edges of complex shapes built from simple geometric objects, such as a cylinder with one end protruding into a sphere. Also further work is being undertaken to include additional electromagnetic objects such as quadrupole and octupole magnets, solenoids, and electric fields of common geometries.

The goal of the expansions is to allow a user to run simulations testing the beam current and focusing for an entire beam line and to test complex, novel magnetic lenses and plasma confinement systems. This will allow users to simulate and optimize their entire apparatus as a whole and add new and/or novel elements to the beam line to test their effect. Also, the added elements will allow for many novel designs of plasma confinement systems to be quickly tested in a standardized format and the results compared on an even basis.

\section{SUMMARY}

We have presented a program written for Mathematica that can conduct automated classical trajectory Monte Carlo simulations of ions in user- 
specified magnetic fields. The user can create a custom magnetic field configuration built from any number of current loops and lines that can be oriented along any of the three coordinate axes. By a similar process, the user can also simulate any number of detectors. Most properties of the simulation can be easily configured, resulting in a generalized tool which can be used to test the focusing and defocusing abilities of magnetic lenses or the quality of confinement in plasma traps.

The program is automated to allow many different parameter configurations of a system to be tested so that new designs can be optimized with little user interaction. This code provides a quick and standard testing tool for novel lenses and plasma traps which can be combined to simulate ion beam lines and other complicated systems. Continued work on the code will provide a more generalized tool including multiple new magnetic components that can be used to model a vast array of experimental configurations

\section{ACKNOWLEDGMENTS}

This material is based upon work supported by the Department of Energy under Grant No. DE-FG0206ER54883 and by the National Science Foundation under Grant No. PHY-1202428.

Computational resources were provided by UNT's High Performance Computing Initiative.

\section{REFERENCES}

1. Mathematica, ver. 8.0.1.0, Wolfram Research Inc., 2010.

2. C. A. Ordonez, "Charged particle transport through a periodic electrostatic potential having a small spatial period," J. Appl. Phys., vol. 104, p. 054903, 2008.

3. Ordonez, C.A., "Drifting Plasma Confinement With A Spatially Periodic Field," IEEE, Trans. Plasma Sci., vol 38, pp. 388-392, 2010.

4. SIMION, ver. 8.1, Scientific Instrument Services, 2012.

5. J. D. Griffiths, Introduction to Electrodynamics, $3^{\text {rd }}$ ed., Prentice-Hall, 1999.

6. J. D. Jackson, Classical Electrodynamics, $3^{\text {rd }}$ ed. Wiley, 1999.

7. J. T. Conway, "Exact Solutions for the Magnetic Fields of Axisymmetric Solenoids and Current Distributions", IEEE, vol. 37, pp. 2977-2988, 2001.

8. R. A. Schill, "General Relation for the Vector Magnetic Field of a Circular Current Loop: a Closer Look," IEEE Trans. On Magnetics, vol. 29, no. 2, pp. 961-967, 2003.

9. L. Uranker, "Vector Potential and Magnetic Field of Current-carrying Finite Arc Segment in Analytical Form, Part I," IEEE Trans. Magn., vol. 16, pp. 12831288, 1980.

10. J. D. Wofford and C. A. Ordonez, "Dual Levitated Coils for Antihydrogen Production," CAARI Conference Proceedings, 2012 